\title{
Placebo-Driven Clinical Trials of Transfer Point Glucan \#300 in Children with Chronic Respiratory Problems: Antibody Production
}

\author{
${ }^{1}$ Vetvicka Vaclav, ${ }^{2}$ Richter Josef, ${ }^{3}$ Svozil Vladimir, \\ ${ }^{2}$ Rajnohova Dobiasova Lucie and ${ }^{2}$ Kral Vlastimil \\ ${ }^{1}$ Department of Pathology, School of Medicine, \\ University of Louisville, Louisville, KY, USA \\ ${ }^{2}$ Zdravotní ústav se sídlem v Ústí nad Labem, Czech Republic, Europe \\ ${ }^{3}$ Sanatorium Edel, Zlaté Hory, Czech Republic, Europe
}

Received 2013-05-17, Revised 2013-05-30; Accepted 2013-06-05

\begin{abstract}
The role of glucan in stimulation of immune reactions has been well-established. In this report, we focused on the antibody production in glucan-supplemented children with chronic respiratory problems. We measured the levels of salivary IgA, IgM and IgG in 40 children aged 8-12 years and evaluated the effects of $100 \mathrm{mg} \mathrm{d}^{-1}$ oral dose of glucan. We found a significant increase in production of all tested antibodies in the glucan-stimulated group, but a decrease of antibody production in the control group. A thirty-day oral application of yeast-based natural immunomodulator $\beta$-glucan strongly stimulated the mucosal immunity of children with chronic respiratory problems.
\end{abstract}

Keywords: Glucan, Children, Mucosal Immunity, IgA, IgM, IgG

\section{INTRODUCTION}

Upper respiratory infections are the highest incidence of acute illness in the developed world. According to the estimates, the average adult has 3 colds per year and the average schoolchild up to 10 (Spector, 1995; Vetvicka and Vetvickova, 2010). Although patients with complications may benefit from antibiotic or inhaler treatment, current medicine has very little to offer for uncomplicated infections. There is no doubt that a need for effective, safe and inexpensive treatment of chronic respiratory problemsexists. Our paper is focused on the natural immunomodulator $\beta$-glucan as a possible solution.

$\beta$-Glucans are well-known natural Biological Response Modifiers (BRM). Thus far, glucans are classified as "non-specific" because their biological effects are pleiotropic. On the other hand, for the past 30 years,
Japan has sucessfully used several forms of mushroomderived $\beta$-glucan in cancer patients (Hamano et al., 1999).

Numerous rodent studies demonstrated that glucan supplements offset the increased risk of infection, usually via stimulation of immunological activities (Davis et al., 2004). In addition, recent findings also showed strong effects on humoral immunity, including antibody formation (Vetvicka and Vetvickova, 2008; 2010). Up to now, the effects of glucan on mucosal immunity has not been studied. Recently, several preclinical and clinical studies showed that yeast-derived glucan reduced respiratory symptoms of women in stress (Talbott and Talbott, 2012) and helped to maintain the body's defense against pathogens (Auinger et al., 2013). For our clinical trial, we used the best characterized insoluble, yeast-derived glucan \#300, which was repeatedly confirmed to be the most active commercially available glucan (Vetvicka and Vetvickova, 2007; 2010). Correspondence Author: Vaclav Vetvicka, Department of Pathology, University of Louisville, 511 S. Floyd, Louisville, KY 40202, USA 
In addition, our previous clinical trials showed a significant increase of salivary lysozyme in glucantreated children (Vetvicka and Vetvickova, 2009). In this report, we used the same model of children with chronic respiratory problems living in an area known for its extremely high level of pollution and focused on the effects of glucan administration on antibody response.

\section{MATERIALS AND METHODS}

\subsection{Protocol}

The same protocol that was previously described (Vetvicka and Vetvickova, 2011) was been used throughout this study. Briefly, a randomized, doubleblind, placebo-controlled trial compared $\beta$-glucan \#300 and placebo in children. Forty children ( 24 females, 16 males, age $8-12$, average $10.7 \pm 2.3$ ) from the sanatorium for respiratory diseases EDEL were enrolled in the 4week trial. The clinical trial was conducted at the Sanatorium EDEL (Zlate Hory, Czech Republic) and the study was approved by the Ethics committees of the Public Health Institute and Sanatorium Edel Czech Republic. This study was performed in agreement with Helsinki declaration (revised version 2000.09.01) and in full compliance with the rules offor clinical testing for the Czech Republic. Parental consent was given in all cases.

Subjects were randomly assigned to groups which were blinded to intervention. During the intervention period, the subjects consumed $100 \mathrm{mg} \mathrm{d}^{-1}$ of $\beta$-glucan or placebo. Both glucan and placebo capsules looked identical. Subjects were routinely evaluated by the medical staff.

\subsection{Glucan}

Yeast-derived insoluble glucan \#300 (>85\% dry w/w basis) was purchased from Transfer Point (Columbia, SC, USA). This glucan contains 96\% carbohydrates and $2.1 \%$ proteins. Neutral sugar analysis confirmed $91.3 \%$ glucose and $8 \%$ mannose.

\subsection{Tests}

In all subjects we obtained saliva at the beginning of the study and at the conclusion of their stay in Sanatorium. We used identical times (between 8 and 9 AM) for sampling, so the possible influence of circadian rhythms could be eliminated.

Saliva was collected using a commercial Salivette device (Sarstead, Orsay, France). After $2 \mathrm{~min}$ of chewing,the cotton swab was added into a sterile container and centrifuged at $1,000 \mathrm{~g}$ for $15 \mathrm{~min}$ and stored at $-15^{\circ} \mathrm{C}$. We measured the levels of sIgA, sIgG and sIgM using nephelometer Siemens BM II as suggested by the manufacturer.

\subsection{Statistical Analysis}

Statistical significance was evaluated by a pair t-test using a GraphPad Prism 502 software (GraphPad Software, USA).

\section{RESULTS}

All children participating in this study are living at the same locality at Nothern Moravia, known for its extremely high level of pollution. Only children diagnosed with repeated upper airway infections, chronic bronchitidis, allergies and/or asthma were used in this study. All subjects were given an identical diet with optimal nutrition and were identically treated using climatotherapie and speleotherapie. In addition, a full medical examination was given at the beginning and end of the trial. The identification of glucan-treated and placebo-treated group was done only on the end of the study.

Our results of the specific antibody response are shown in Table 1. Our data suggest that at the beginning of the study, the levels of IgG were identical in both groups. However, the glucan-supplemented group showed a strong increase in IgG production, whereas placebo group expressed significant decrease of salivary IgG levels (Fig. 1).

Similar dynamics were found in formation of salivary IgA and iGM, where the glucan supplementation caused strong increase, whereas control group showed significant decrease in both $\operatorname{SIgA}$ and IgM values (Fig. 2 and 3). The highest increase was found in $\operatorname{sigA}(183 \%)$.

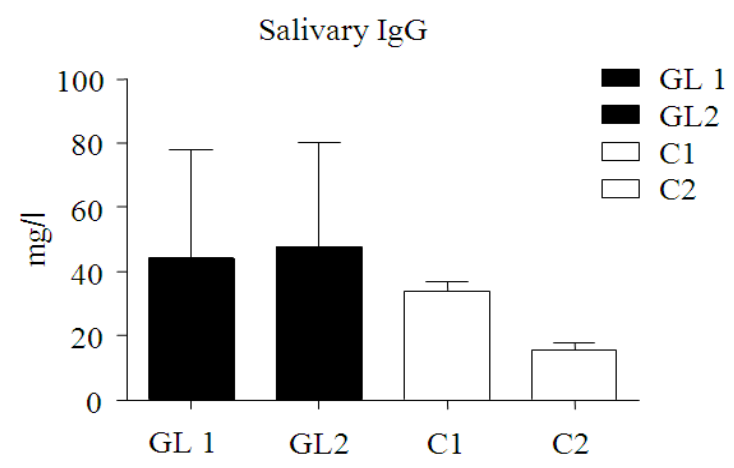

Fig. 1. Effects of 4-week oral administration of glucan (100 $\mathrm{mg}$ day $^{-1}$ ) on $\mathrm{IgG}$ levels in saliva. GL 1 represents glucan group at day 1; GL 2 at day 30. C1 represents control (placebo) group at day 1, C2 at day 30 . Significant at $\mathrm{p}<0.05$ between groups 


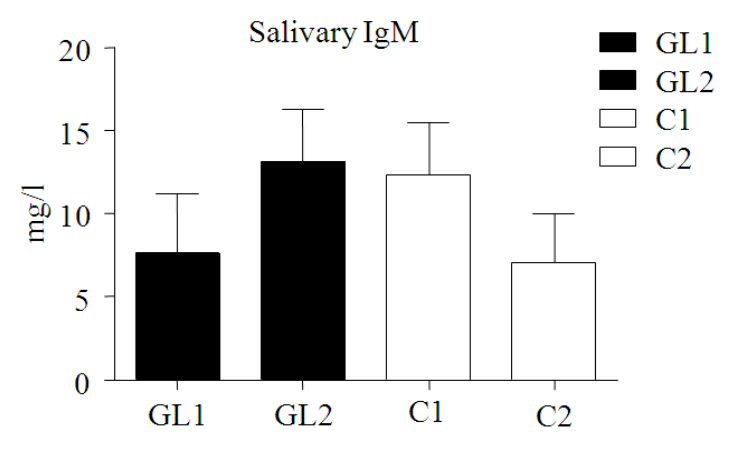

Fig. 2. Effects of 4-week oral administration of glucan $(100 \mathrm{mg}$ day $^{-1}$ ) on IgM levels in saliva. GL 1 represents glucan group at day 1; GL 2 at day 30. C1 represents control (placebo) group at day $1, \mathrm{C} 2$ at day 30 . Significant at $\mathrm{p}<0.05$ between groups

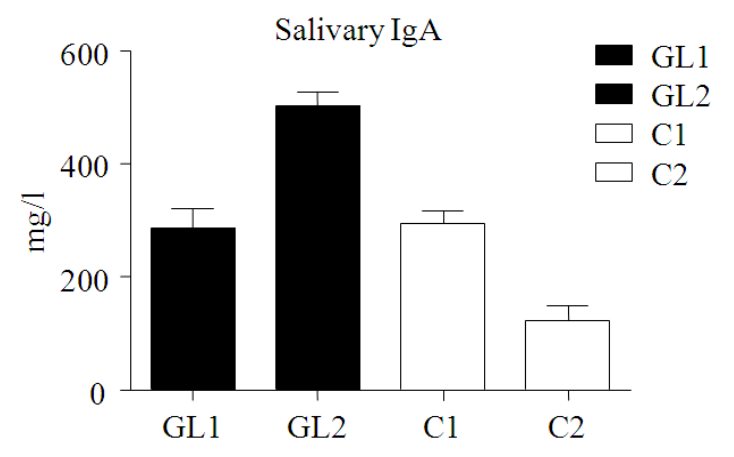

Fig. 3. Effects of 4-week oral administration of glucan (100 mg day $^{-1}$ ) on IgA levels in saliva. GL 1 represents glucan group at day 1; GL 2 at day 30. C1 represents control (placebo) group at day $1, \mathrm{C} 2$ at day 30 . Significant at $\mathrm{p}<0.05$ between groups

Table 1. Mean concentration of $\operatorname{IgA}$, IgG and IgM saliva of children at baseline (day 1) and after completion of oral administration of glucan (day 30 )

\begin{tabular}{lllll}
\hline & GL1 & GL2 & C1 & C2 \\
\hline $\operatorname{IgG~(mg/l)~}$ & $44,3(33,6)$ & $47,4(32,3)$ & $33,9(2,9)$ & $15,5(2,4)$ \\
& $\mathrm{p}<0,0001$ & & $\mathrm{p}<0,0001$ & \\
$\operatorname{IgA}(\mathrm{mg} / \mathrm{l})$ & $288,4(30,8)$ & $502,9(26,0)$ & $295,1(23)$ & $123,0(24)$ \\
& $\mathrm{p}<0,0001$ & & $\mathrm{p}<0,0001$ & \\
$\operatorname{IgM}(\mathrm{mg} / 1)$ & $7,6(3,6)$ & $13,2(3,1)$ & $12,3(3,2)$ & $7,1(2,9)$ \\
& $\mathrm{p}<0,001$ & & $\mathrm{p}<0,001$ & \\
\hline
\end{tabular}

GL 1 represents glucan group at day 1; GL 2 at day $30 . \mathrm{C} 1$ represents control (placebo) group at day $1, \mathrm{C} 2$ at day 30

\section{DISCUSSION}

Salivary anti-microbial systems are usually divided into immune (secretory antibodies) and non- immunoglobulin (lysozyme, agglutinins, lactoferrin). Most children with respiratory problems show a normal immunological status (Hosking et al., 1978), probably with exception of IgA (Ostergaard, 1980). Additional reports showed serum IgG and IgM levels to be significantly elevated, with sIgA remarkably low, suggesting the important role of salivary IgA in fighting respiratory infections (Lehtonen et al., 1987). Children living permanently in a region with a high level of pollution were found to have increased levels of sIgA (particularly in children with high frequency of respiratory diseases), which is usually increased after several weeks in nonpolluted areas (Richter and Pelech, 1996).

After the early research on mucosal immunity, recent decades show a revival of interest in salivary proteins in clinical immunology (Ambatipudi et al., 2009; Castagnola et al., 2011; Fabian et al., 2012). Some of the reasons might be the non-invasiveness of saliva collection, the lack of need of trained personnel and the lack of a risk of infection. Saliva is often considered to be a "body mirror" with little used abilities in diagnostics (Zhang et al., 2009). Proteomic studies lately helped to overcome most of the criticism suggesting that the saliva content is influenced by either lifestyle, genetic markup, age or sex (Pfaffe et al., 2011; Pink et al., 2009). Today we are able to find in saliva over 2,400 components, whereas only $20 \%$ of them can be found in plasma (Ambatipudi et al., 2009; Castagnola et al., 2011; Pfaffe et al., 2011; Lehne et al., 2006; Topkas et al., 2012). Our laboratory has been specializing in salivary immunity for quite some time for both clinical evaluation and for studies of the effects of environmental pollution on large populations. The current use of new Salivette devices significantly increased the quality of results (Topkas et al., 2012).

For our study we choose glucan \#300, which is currently not only highly studies glucan, but also a glucan with the highest and most pleiotropic activities (Vetvicka and Vetvickova, 2010; 2012) regardless the rout of administration (Vetvicka and Vetvickova, 2010). This study is a follow-up of our original finding of the effects of this particular glucan on secretory proteins in saliva, including CRP and lysozyme (Vetvicka and Vetvickova, 2011). Again, we tested the effects of glucan supplementation on children living in region with extreme environment pollution and suffering from chronic respiratory problems.

Properly functioning immune system is crucial to defend against all invading pathogens, including common cold viruses. Currently, yeast-derived glucans 
were confirmed to have beneficial effects against common cold and respiratory symptoms (Talbott and Talbott, 2012; Auinger et al., 2013) and against allergy symptoms via decreasing proinflammatory cytokines such as IL- 6 and TNF- $\alpha$ and increasing of secretion of IL10 and accession of cellular antioxidants (Senoglu et al., 2008). These studies, however, were restricted to adults. In addition, glucan was found to significantly improve the immunosuppression caused by various toxins (Vetvicka and Vetvickova, 2012), making it an optimal molecule for children from environmentally poluted areas.

Whereas immunomodulatory effects of glucan in both animal models and clinical studies are well established (Novak and Vetvicka, 1987; Ramberg et al., 2010), very little is known about the effects on salivary immunity (Lehne et al., 2006). No clinical study evaluating glucan affecting antibody response of children with respiratory problems exists in the literature.

sIgA represents the most studied salivary component. Studies on dogs (Stuyven et al., 2010) and humans (Lehne et al., 2006) demonstrated fast and significant increase of sIgA levels. Different situation was found in case of sIgG: an increase in dogs (Stuyven et al., 2010), but no changes in humans (Lehne et al., 2006). Our data showing strong parallel increases in the levels of both sIgA and sIgG suggest possible effects of dose and the quality of glucan. Some suggest that the correlation of levels of salivary $\operatorname{IgA}$ and $\operatorname{IgG}$ are in inflammation influenced by permeability of capillaries (Gleeson et al., 1995), others hypothesize the effects of stress factors (Ambatipudi et al., 2009; Brown et al., 1977; Francis et al., 2002), which cannot be overlooked in our model of children during their early days at the sanatorium. Low IgG level in control group (C2) correlates with albumin levels (Vetvicka and Vetvickova, 2011). Decrease in albumin level (as indicator of inflammation) is in compromised children caused by reduction of mucosal irritation in respiratory tract, which is correlated by glucan-induced stimulation of IgG production.

The findings of increased levels of sIgM just add another stone to the mosaic of glucan-mediated immune response in disease-prone children. The increase of sIgM after glucan supplementation correspond to (Stuyven et al., 2010), the higher levels in control group (C1) were most probably caused by sublinical infection in some children, which in a relatively low number of samples could significantly influence the average amount. Our data also showed significant improvement of the overall health of tested children.

\section{CONCLUSION}

Taken together, our results strongly suggest that natural immunomodulator glucan can significantly improve the health of children with chronic respiratory problems, probably via stimulation of their mucosal immunity.

\section{ACKNOWLEDGEMENT}

This study was supported by Technology Agency of the Czech Republic TACR TA 0202094.

\section{REFERENCES}

Ambatipudi, K.S., B. Lu, F.K. Hagen, J.E. Melvin and J.R. Yates, 2009. Quantitative analysis of age specific variation in the abundance of human female parotid salivary proteins. J. Proteome Res., 8: 50935102. DOI: 10.1021/PR900478H

Auinger, A., L. Rieda, G. Bothe, R. Busch and J. Gruenwald, 2013. Yeast (1,3)-(1,6)-beta-glucan helps to maintain the body's defence against pathogens: A double-blind, randomized, placebocontrolled, multicentric study in healthy subjects. Eur. J. Nutr. DOI: 10.1007/S00394-013-0492-Z

Brown, L.R., W.J. Frome, M.G. Wheatcroft, L.J. Riggan and N.E. Bussell et al., 1977. Clinical and materials sciences the effect of skylab on the chemical composition of saliva. J. Dent. Res., 1: 1137-1143. DOI: 10.1177/00220345770560100101

Castagnola, M., P.M. Picciotti, I. Messana, C. Fanali and A. Fiorita et al., 2011. Potential applications of human saliva as diagnostic fluid. Acta. Otorhinolaryngol. Ital., 31: 347-357. PMID: 22323845

Davis, J.M., E.A. Murphy, A.S. Brown, M.D. Carmichael and A. Ghaffar et al., 2004. Effects of moderate exercise and oat $\beta$-glucan on innate immune function and susceptibility to respiratory infection. Am. J. Physiol. Regul. Integr. Comp. Physiol., 286: 366-372. DOI: 10.1152/AJPREGU.00304.2003

Fabian, T.K., P. Hermann, A. Beck, P. Fejerdy and G. Fabian, 2012. Salivary defense proteins: Their network and role in innate and acquired oral immunity. Int. J. Mol. Sci., 13: 4295-4320. DOI: 10.3390/IJMS13044295

Francis, J.L., M. Gleeson, D.J. Lugg, R.L. Clancy and J.M. Ayton et al., 2002. Trends in mucosal immunity in Antarctica during six Australian winter expeditions. Immunol. Cell Biol., 80: 382-390. DOI: 10.1046/J.1440-1711.2002.01104.X 
Gleeson, M., A.W. Cripps and R.L. Clancy, 1995. Modifiers of the human mucosal immune system. Immunol. Cell Biol., 73: 397-404. DOI: 10.1038/ICB.1995.62

Hamano, K., H. Gohra, T. Katoh, Y. Fujimora and N. Zempo et al., 1999. The preoperative administration of lentinan ameliorated the impairment of natural killer activity after cardiopulmonary bypass. Int. J. Immunopharmacol., 21: 531-540. DOI: 10.1016/S0192-0561(99)00033-8

Hosking, C.S., M.G. Fitzgerald and M.J. Shelton, 1978. The immunological investigation of children with recurrent infections. Aust Paediatr. J. PMID: 105699

Lehne, G., B. Haneberg, P. Gaustad, P.W. Johansen and H. Preus et al., 2006. Oral administration of a new soluble branched $\beta-1,3-\mathrm{D}$-glucan is well tolerated and can lead to increased salivary concentrations of immunoglobulin A in healthy volunteers. Clin. Exp. Immunol., 143: 65-69. DOI: 10.1111/J.13652249.2005.02962.X

Lehtonen, O.P.J., J. Tenovuo, A.S. Aaltonen and P. Vilja, 1987. Immunoglobulins and innate factors of immunity in saliva of children prone to respiratory infections. Acta. Pathol. Microbiol. Immunol. Scand. Sect. C: Immunol., 95C: 35-40. DOI: 10.1111/j.1699-0463.1987.tb00006.x

Novak, M. and V. Vetvicka, 1987. Glucans as biological response modifiers. Endocrine Metabol. Immune Disorders, 9: 67-75. DOI: 10.2174/187153009787582423

Ostergaard, P.A., 1980. Clinical and immunological features of transient IgA deficiency in children. Clin. Exp. Immunol., 40: 561-565. PMID: 7418266

Pfaffe, T., J. Cooper-White, P. Beyerlein, K. Kostner and C. Punyadeera, 2011. Diagnostic potential of saliva: Current state and future applications. Clin. Chem., 57: 675-687. DOI: 10.1373/CLINCHEM.2010.153767

Pink, R., J. Simek, J. Vondrakova, E. Faber and P. Michl et al., 2009. Saliva as a diagnostic Medium. Biomed. Pap. Med. Fac. Univ. Palacky Olomouc Czech Repub., 153: 103-110. DOI: 10.5507/BP.2009.017

Ramberg, J. E., E.D. Nelson and A. Sinnott, 2010. Immunomodulatory dietary polysaccharides: A systematic review of the literature. Nutrition J., 9: 54-76. DOI: 10.1186/1475-2891-9-54

Richter, J. and L. Pelech, 1996. Immunological findings in groups of children after compensatory measures. Toxicol. Lett., 88: 165-168. DOI: 10.1016/03784274(96)03733-2
Senoglu, N., M.F. Yuzbasioglu, M. Aral, M. Ezberci and E.B. Kurutas et al., 2008. Protective effects of Nacetylcysteine and $\beta$-glucan pretreatment on oxidative stress in cecal ligation and puncture model of sepsis. J. Invest. Surg., 21: 237-243. DOI: 10.1080/08941930802180136

Spector, S.L., 1995. The common cold: Current therapy and natural history. J. Allergy Clin. Immunol., 95: 1133-1138. DOI: 10.1016/S0091-6749(95)70218-0

Stuyven, E., F. Verdonck, I.V. Hoek, S. Daminet and L. Duchateau et al., 2010. Oral administration of $\beta$ 1,3/1,6-glucan to dogs temporally changes total and antigen-specific $\operatorname{IgA}$ and $\operatorname{IgM}$. Clin. Vaccine Immunol., 17: 281-285. DOI: 10.1128/CVI.0034409

Talbott, S.M. and J.A. Talbott, 2012. Baker's yeast betaglucan supplement reduces upper respiratory symptoms and improves mood state in stressed women. J. Am. Coll. Nutr., 31: 295-300. PMID: 23378458

Topkas, E., P. Keith, G. Dimeski, J. Cooper-White and C. Punyadeera, 2012. Evaluation of saliva collection devices for the analysis of proteins. Clin. Chim. Acta, 413: 1066-1070. DOI: 10.1016/J.CCA.2012.02.020

Vetvicka, V. and J. Vetvickova, 2007. Physiological effects of different types of $\beta$-glucan. Biomed. Pap. Med. Fac. Univ. Palacky Olomouc Czech Repub., 151: 225-231. DOI: 10.5507/BP.2007.038

Vetvicka, V. and J. Vetvickova, 2008. A comparison of injected and orally administered $\beta$-glucans. J. Am. Nut. Assoc., 11: 42-48.

Vetvicka, V. and J. Vetvickova, 2009. Effects of yeastderived $\beta$-glucans on blood cholesterol and macrophage functionality. J. Immunotoxicol., 6: 3035. DOI: $10.1080 / 15476910802604317$

Vetvicka, V. and J. Vetvickova, 2010. $\beta-1,3-$-Glucan: Silver bullet or hot air? Open Glycosci., 3: 1-6.

Vetvicka, V. and J. Vetvickova, 2011. $\beta(1-3)$-D-glucan affects adipogenesis, wound healing and inflammation. Orient. Pharm. Exp. Med., 11: 169175. DOI: $10.1007 /$ S13596-011-0024-4

Vetvicka, V. and J. Vetvickova, 2012. Glucanresveratrol-vitamin $\mathrm{C}$ combination offers protection against toxic agents. Toxins, 4: 1301-1308. DOI: 10.3390/TOXINS4111301

Zhang, L., H. Xiano and D.T. Wong, 2009. Salivary biomarkers for clinical applications. Mol. Diagn. Ther., 13: 245-259. DOI: 10.1007/BF03256330 